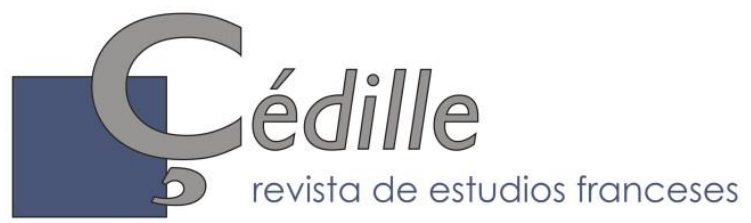

ISSN: 1699-4949

$n^{0} 18$ (otoño de 2020)

Monografías 11

Epistemocrítica: análisis literario y saber científico

Amelia Gamoneda Lanza \& Francisco González Fernández, editores científicos

\title{
Perception et mouvement dans la poésie de Gilles Cyr
}

\author{
Víctor BERMÚDEZ \\ Universidad de Salamanca \\ victorbermudez@usal.es \\ ORCID: 0000-0002-9548-7290
}

\section{Resumen}

El estudio aborda el universo sensorial de la poesía de Cyr a través de los paradigmas de la neurofisiología de la percepción visual y motora y de la fenomenología del movimiento. Concretamente, las nociones de «perceptos visuales» y «representaciones visuomotoras» se examinan en el texto atendiendo a las singularidades específicas del lenguaje poético de Gilles Cyr, donde la relación del cuerpo con lo desconocido también ocupa un lugar relevante. Del mismo modo, la distinción entre «visión epistémica» y «noepistémica» se tiene en cuenta a lo largo de este trabajo. El análisis considera las reflexiones de la crítica literaria relativas al tratamiento del espacio y del movimiento tanto como al nivel epistemológico de esta poesía.

Palabras clave: Teoría de la percepción, Poesía quebequense, Poética cognitiva.

\section{Résumé}

L'étude aborde l'univers sensoriel de la poésie de Cyr au moyen des paradigmes de la neurophysiologie de la perception visuelle et motrice et de la phénoménologie du mouvement. Plus précisément, les notions de «percepts visuels » et de «représentations visuomotrices » seront examinées à partir du texte littéraire afin d'expliquer certaines singularités du langage poétique cyrien, où la relation du corps avec l'inconnu occupe également une place. De même, la distinction entre «vision épistémique » et «non épistémique » sera prise en compte tout au long de ce travail. L'analyse reprend les réflexions de la critique littéraire sur l'écriture cyrienne concernant le traitement de l'espace et du mouvement, et aussi ceux qui concernent le niveau épistémologique de cette écriture.

Mots clé: Théorie de la perception, Poésie québécoise, Poétique cognitive.

\begin{abstract}
The study addresses the sensory universe of Cyr's poetry through the paradigms of neurophysiology of visual and motor perception and the phenomenology of movement. More specifically, the notions of "visual percepts" and "visuomotor representations" are examined in the text according to the specific singularities of the poetic language of Gilles Cyr, where the relationship of the body with the unknown also occupies a relevant place. Similarly, the distinction between "epistemic vision" and "non-epistemic" is taken
\end{abstract}

*Artículo recibido el 25/02/2020, aceptado el 25/10/2020. 
into account throughout this study. The analysis takes into account the reflections of literary criticism related to the treatment of space and movement, as well as the epistemological level of this poetry.

Keywords: Theory of perception. Québec poetry. Cognitive Poetics.

\section{Introduction : Gilles Cyr et l'épistème en mouvement}

Gilles Cyr est né à Saint-Fidèle-de-Ristigouche (Gaspésie) en 1940. En guise de contextualisation biographique, on peut noter qu'il a suivi des études de Licence ès lettres (1970) et une Maîtrise ès lettres (1971) à l'Université de Montréal, ainsi que des séminaires de doctorat ès lettres à l'Université de Paris-VII (1971-1973). Il a été chargé de cours de littérature québécoise et française à l'Université de Thunder Bay (Ontario, 1974), à l'Université du Québec à Montréal, (1975-1977), à l'Université Western Ontario, London (1976), à l'Université Laval (1977), à l'Université de Montréal (1977-1979) et à l'Université Concordia (1978). Dans le domaine de l'édition, il a été conseiller littéraire aux Éditions de l'Hexagone $^{1}$ (1977-2007) où il a également dirigé la collection historique « Rétrospectives » (2003-2007), qui a commencé par la publication de L'Âge de la parole (1965, réédité en 2013) de Roland Giguère. Parmi les prix récompensant son œuvre littéraire, on peut citer le Prix du Gouverneur général (dans la section poésie, 1992), la Bourse d'Écriture Gabrielle-Roy (1999), le Prix de l'Institut Coréen de Traduction Littéraire (également, dans la section poésie, avec Han Daekyun, 2001) et le Prix Arthur-Buies (2010). En outre, de nombreux voyages et séjours internationaux ont marqué la trajectoire et les collaborations de Gilles Cyr avec d'autres artistes, tels que Viviane Prost, Renée Lavaillante ou Bertrand Bracaval ; finalement Cyr a également développé une activité en tant que traducteur de poètes coréens, perses et arméniens, à laquelle il a parallèlement consacré des travaux relatifs à la poétique de l'acte de traduction.

\footnotetext{
${ }^{1}$ Ayant pris son nom à partir de leurs six membres fondateurs (Gaston Miron, Gilles Carle, JeanClaude Rinfret, Olivier Marchand, Mathilde Ganzini et Louis Portugais) l'Hexagone est, depuis sa fondation en 1954 et jusqu'à nos jours, une des principales maisons d'édition de poésie au Canada et elle va centraliser une grande partie du catalogue poétique québécois. Comme l'ont remarqué les historiens et critiques littéraires Laurent Mailhot et Pierre Nepveu dans leur anthologie La Poésie québécoise (1996) : «Beaucoup plus qu'une simple maison d'édition, l'Hexagone a été un centre d'animation, un lieu idéologique et, en définitive, le foyer de toute une génération de poètes, même de ceux qui n'y ont pas publié leurs livres [...]» (21).

Cet article a été réalisé dans le cadre des activités financées par le Projet I+D d'excellence Inscripciones literarias de la ciencia: cognición, epistemología y epistemocrítica du Ministère espagnol de l'Économie et de la Compétitivité (réf. : FFI2017-83932-P). Du même, cette étude a compté avec le soutien du Centre de recherche sur le texte et l'imaginaire - FIGURA (Université du Québec à Montréal) et du Dr. Marc-André Brouillette. Le poète Gilles Cyr a eu la gentillesse et de me permettre de travailler dans ses archives personnelles, et de m'accorder un entretien (Bermúdez, 2019) pendant le processus de recherche de cet article.
} 
Par ailleurs, l'œuvre de Gilles Cyr témoigne d'une exploration du langage qui exprime une tension entre le corps et l'espace ${ }^{2}$. Une telle enquête permet de concevoir cette poésie comme une écriture qui s'approche de la représentation de l'extérieur en tendant les articulations entre sujet et objet, car la tendance de l'individu vers le monde qu'élabore l'écriture cyrienne est une inclination tendue : le poème est un tenseur. À l'intérieur de cette tension, deux types de connaissances se rencontrent : un savoir dit épistémique et un autre que l'on décrira provisoirement comme phénoménologique. En conséquence, cette poétique elliptique et par moments hermétique condense une connaissance dérivée de la volonté de catégorisation du réel qui est «propre du sujet », et une autre qui découle du processus de se situer comme un corps dans le monde ; mais un corps qui a la volonté de représenter.

Il est pertinent de souligner que l'exploration formelle de la poétique cyrienne évolue de manière très significative. À titre d'exemple, dans l'édition 2010 de Brouillette qui réunit Sol inapparent (1978), Diminution d'une pièce (1983) et Songe que je bouge (1994) - ${ }^{\circ}$ dont la première section s'intitule précisément « connaissance »- se produit un changement abrupt par rapport aux éditions précédentes en ce qui concerne la disposition des poèmes dans la page. Les poèmes de cette édition apparaissent groupés par paires, ce qui fait associer, dans une même unité de sens, deux textes qui étaient originalement disposés dans des pages différentes, modifiant ainsi la nature même de l'expression car chaque page devient plus fluide. Ainsi, l'on trouve une confection moins elliptique et une réduction de la tension visuelle portée par le blanc de la page qui été caractéristique des premiers recueils de Cyr.

Ceci coïncide également avec l'évolution formelle des livres les plus récents de Cyr. Cette transformation qui va du «serré » vers «le fil de la page » montre sa plus claire expression dans des recueils tels que Fruits et frontières (2006) ou Huit sorties (2012) tandis qu'elle est graduelle et s'observe déjà dans Erica je brise (2003). À titre comparatif, il faut remarquer que l'évolution de la poésie cyrienne tend, du point de vue formel, à s'allonger progressivement par rapport à la concision extrême de ses premiers recueils, partagée avec d'autres auteurs québécois comme Pierre Labarge - L'œil de nuit (1973), Dedans dehors (1977), Vue du corps (1979) - mais aussi

\footnotetext{
${ }^{2}$ Comme on le remarquera plus tard, la question de l'espace constitue un des sujets primordiaux de la littérature québécoise. À titre d'exemple, l'on peut citer l'œuvre de Saint-Denys Garneau (1912-1943), Regards et jeux dans l'espace (1937, réédité en 2000). Garneau, une des figures historiques de la littérature québécoise, montre dès sa jeunesse un intérêt pour la photographie et la peinture. Regards et jeux dans l'espace, son seul recueil de poésie, explore les relations de l'espace et la vision, tout en interpellant ses contemporains: «vous ne savez pas jouer avec l'espace» (Garneau, $2000: 19$ ). La corporalité se traduit par toute une nomenclature du corps en train de regarder («visage », « yeux », « gestes ») et par une nomination de la spatialité enrichie par le comportement du monde extérieur («paysage », « oiseaux », « arbres »), envisageant ainsi une représentation de l'équilibre du regard, au sein duquel convergent des ondulations, la transparence, et la couleur où « la danse est paraphrase de la vision» (Garneau, $2000: 20)$.
} 
avec des écrivains français tels que Jacques Dupin - notamment dans ses premiers livres de poésie, Gravir (1963), L'embrasure (1969), Dehors (1975) ou Une apparence de soupirail (1982), regroupés ultérieurement dans Le corps clairvoyant (2013) -, ou celle d'André du Bouchet - Air (1951), Dans la chaleur vacante (1959), Ou le soleil (1968) - ou celle encore de Lorand Gaspar - Le quatrième état de la matière (1966), Gisements (1968), Sol absolu (1972) et encore Corps corrosifs (1978) -. Délaissant peu à peu cette concision, la poésie cyrienne se rapproche alors des structures plus caractéristiques de l'écriture fluide d'un Bernard Noël - La Face de silence (1967), La Moitié du geste (1982) ou Les Yeux dans la couleur (2004) ou d'un Jacques Ancet - Sous la montagne (1992), La Brûlure (2002) -, pour donner quelques exemples contemporains en France. De même, dans la tradition québécoise, Fernand Ouellette - Ces anges de sang (1955), Le Soleil sous la mort (1965), Dans le sombre (1967), Ici, ailleurs, la lumière (1977) -, Robert Melançon - Peinture aveugle (1978), Le paradis des apparences (2004) - ou Michel Van Schendel - De l'ceil et de l'écoute (1980) - se trouvent dans la constellation littéraire qui donne sens, soit par la différenciation et la distanciation, soit par l'accompagnement, à l'œuvre de Gilles Cyr.

Comme on l'a déjà noté, au cours de la trajectoire littéraire de Gilles Cyr, plusieurs types de savoirs convergent dans sa poétique. Cependant, avant de se pencher sur les mouvements de la connaissance dans le langage poétique, un bref parcours à travers les contacts entre le langage scientifique et le littéraire semble nécessaire, et fera l'objet du point suivant de ce travail «Autour de la science dans l'expression lyrique québécoise ». En effet, l'intérêt de Gilles Cyr pour le domaine scientifique se manifeste dès sa jeunesse intellectuelle, quand, dans une tentative de rapprochement avec la théorie littéraire, il écrit l'article « Littérature et science », publié dans la revue Liberté en $1970^{3}$. Cet essai questionne les niveaux de réalité et les notions du réel que la science et la littérature engagent. Science et littérature, toutes deux, sont ici conçues comme générateurs de modes spécifiques du réel. La réflexion centrale de l'article est celle des méthodes de production de la réalité ; et la ligne qu'elles suivent, une ligne évolutive (pour la science) et discontinue (pour la littérature). Pour Cyr, l'impact que la science a sur la réalité passe par une profonde rénovation de la technique qui modifie les pratiques quotidiennes, alors que celui qu'a la littérature passe par sa rénovation du langage, en s'approchant ainsi de l'idéologie. Dans ce contexte, la nature transformatrice de la littérature s'impose comme une structure de pensée enracinée dans une subjectivité qui lui est propre : «Le rôle de la littérature n'est plus d'interpréter directement le monde, mais de voir comment les langages l'interprètent », remarque Gilles Cyr (1970:63). On constate, dans cet article, un traitement primordialement philosophique (notamment fondé sur Ludwig Wittgenstein, Roland Barthes, Michel Foucault, Aldous Huxley) mais aussi sémio-

\footnotetext{
${ }^{3}$ Fondée en 1959, la revue Liberté est une salle de débat dans le milieu littéraire québécois, de discussion et d'expérimentation sur la poésie de l'époque, où on retrouve les écrivains de la génération dite «L'Hexagone » explorant des registres essayistes et critiques, esthétiques ou politiques.
} 
tique (Julia Kristeva) de la problématique, où Gilles Cyr avance que c'est au sein de la notion de "résultat » que réside la possibilité d'un rapprochement entre la science et la littérature. En outre, Cyr en vient à voir la métaphore comme un point d'articulation des processus de raisonnement scientifique et littéraire, concernant la capacité de la science à déployer des modèles du réel dans la « conjecture ", c'est-à-dire, dans sa prospection des structures du monde. Il anticipe également que la plus grande recherche à mener - à l'époque de la publication du texte, en 1970 - porte sur la nature des langages des deux domaines. Il s'agit d'examiner les savoirs et les incertitudes engendrées par les restrictions des langages eux-mêmes.

$\mathrm{Au}$ sein du langage littéraire résident différents types de connaissance. Comme on le verra, la poésie de Cyr mobilise des approches par moments volontairement subjectives et épistémiques. Son écriture élabore une démarche d'exploration de l'espace exprimée par un langage serré, dans une première étape, exemplifiée par des recueils tels que Sol inapparent (1978) et Diminution d'une pièce (1983), et plus fluide dans un deuxième moment, comme dans Fruits et frontières (2006) et Huit sorties (2012). Les travaux de Marc-André Brouillette constituent une des références les plus fondamentales de la critique littéraire cyrienne. Notamment, son étude Spatialité textuelle dans la poésie contemporaine (2010) ainsi que le dossier qu'il a dirigé pour la revue Voix et Images (2003) consacré à l'œuvre du poète québécois. Brouillette (2010 : 53) remarque que :

La poésie de Gilles Cyr propose un regard désireux de saisir et d'ordonner le monde. Devant le caractère souvent étrange des événements extérieurs, le poète apparait comme un observateur intrigué par la nature et les engrenages d'un tel spectacle. Si la perception qu'on peut avoir de ces événements peut sembler parfois brouillée et confuse, la poésie devient un moyen de configurer un ordre permettant de distinguer, de séparer et d'associer des éléments qui autrement demeureraient indiscernables.

Brouillette propose une étude exhaustive de nature philologique avec une visée phénoménologique à propos des différents niveaux du langage poétique de Gilles Cyr. On reviendra plus tard sur les dimensions phénoménologiques de l'écriture cyrienne en les analysant depuis la perspective de la poétique cognitive. Cependant, il convient de noter maintenant que la poésie cyrienne a également fait l'objet d'approches épistémocritiques qui ont examiné les fonctions littéraires de la connaissance scientifique à l'intérieur de l'œuvre littéraire. Dans ce cadre, des notions épistémiques propres à la poétique de Gilles Cyr ont été analysées à partir de concepts tels que de la «vérité scientifique » et la «vérité lyrique »; tout comme les rapports entre «l'intertextualité » et «l'interdiscursivité »dans cette poésie. Les travaux les plus exhaustifs à cet égard ont été développés par le critique littéraire Jacques Paquin, comme on le montrera ensuite ; il suffit pour le moment de signaler qu'une telle poétique demande une approche qui réponde simultanément à ses caractéristiques épistémiques et phénoménologiques. En ce 
sens, cet article propose une méthode d'étude du point de vue de la poétique cognitive, fondée sur des travaux précédents concernant la poésie d'expression française et espagnole (voir Bermúdez, 2015, 2017, 2019). Ainsi, au vu des propos théoriques et analytiques de ce travail, l'on propose d'approcher la poétique cyrienne à partir de la notion d' « épistème en mouvement », qui sera ultérieurement illustrée.

Ainsi, cette étude aborde l'univers sensoriel de la poésie de Cyr au moyen des paradigmes de la neurophysiologie de la perception visuelle et motrice et de la phénoménologie du mouvement. Plus précisément, les notions de «percepts visuels » (visual percepts) et de «représentations visuomotrices » (visuomotor representations) seront examinées à partir du texte littéraire afin d'expliquer certaines singularités du langage poétique cyrien, où l'inconnu occupe également une place. De même, la distinction entre « vision épistémique » et «non épistémique » observée par Pierre Jacob et Marc Jeannerod sera prise en compte tout au long de ce travail. L'analyse reprend les réflexions de la critique littéraire sur l'écriture cyrienne concernant le traitement de l'espace et du mouvement, mais également en ce qui concerne le niveau épistémologique de cette écriture. On soutiendra que les composantes perceptuelles d'œuvres telles que Sol inapparent (1978) ou Diminution d'une pièce (1983), entre autres, oscillent entre les contenus conceptuels et non conceptuels, et l'on examinera leurs rôles poétiques.

Pour ce faire, nous devrons entrer dans les représentations iconiques de l'œuvre, dans la représentation du corps et du mouvement et dans l'espace. Le paysage, les objets et le sujet seraient configurés dans cette écriture à partir d'une tension du statique et du dynamique. Une telle tension, déjà évoquée, atteint la forme du langage, en particulier la syntaxe et l'analogie, où la figure de la juxtaposition devient la procédure principale de versification de cette «épistème en mouvement ».

\section{Autour de la science dans l'expression poétique québécoise}

Parmi les nombreuses études fondatrices de la discipline épistémocrique qui se sont développées au Québec - dont les travaux de Michel Pierssens et JeanFrançois Chassay, en particulier -, l'on doit mettre l'accent sur celles, plus récentes, du critique littéraire Jacques Paquin. Paquin a fourni un travail essentiel au niveau des intersections entre la science et la poésie dans le contexte québécois, et il a été en charge de l'édition de l'Anthologie Science et Poésie (2014) qui offre un parcours à travers la poésie québécoise du XXe siècle selon son dialogue avec la connaissance scientifique, notamment au travers de l'œuvre de poètes tels que d'Édouard-Zotique Massicotte, Maude Smith-Gagnon, Nicole Brossard, Jocelyne Felx, Gérald Godin, Madeleine Gagnon, Gilles Hénault, Gatien Lapointe, PaulMarie Lapointe ou Claude Péloquin, sans oublier Gilles Cyr. Dans ce travail, Jacques Paquin montre la prédominance des savoirs de la botanique (Lapointe), de l'histoire naturelle (Renaud, Longchamps, Paul Morin), ou de la géologie (Camille Laverdière) dans la littérature québécoise.

Cependant, le critique ne se borne pas à tracer un parcours historique des 
relations que la poésie québécoise établit, à divers degrés, avec la science : il questionne aussi le statut épistémique du savoir scientifique inscrit dans le langage littéraire. Le résultat de ce travail anthologique met en évidence l'immense recherche qui reste encore à faire dans l'élaboration d'une taxonomie qui structure la circulation des savoirs dans la littérature québécoise en fonction des ressources, des intensités, des affinités, ainsi que des «co-dépendances » science-poésie. De même, il ressort des nombreux exemples que la connaissance scientifique justifie l'inscription des textes dans un cadre plus ample d'explorations géo-poétiques, où la nature du pays devient un objet d'attention pour les scientifiques et pour les écrivains, souvent relié aussi à la construction de l'identité. Comme l'a souligné Claude Beausoleil (1996: 7), « la poésie québécoise est née de la nécessité de dire sa différence et son être singulier. L'Amérique est son cadre et ses racines; l'Europe, son origine $»^{4}$; et un tel besoin ne peut qu'influencer les taxonomies de la science québécoise récente.

On trouvera dès lors particulièrement intéressante, par exemple, la fonction de la botanique dans la caractérisation de la nature mettant au jour les spécificités du paysage québécois, ainsi que l'usage d'une poésie «botaniquement informée » qui articule une vision de la culture et de l'identité québécoises, qui viennent toutes les deux enrichir la nomenclature scientifique du français canadien au Québec. Pour sa part, la recherche sur la fonction de la poésie est précisément un des sujets clés de la tradition lyrique québécoise, approfondi notamment entre 1945 et 1970, période d'émancipation non seulement des écrivains mais également des maisons d'édition québécoises qui, pendant la Seconde Guerre mondiale, se sont développées ou consolidés au Québec, comme l'a souligné François Dumont dans son ouvrage Usages de la poésie (1993), ouvrage de référence dans l'histoire de la littérature québécoise. Philosophie, science et société convergent dans les explorations de la poésie québécoise de cette période. Comme l'a remarqué l'historien de la littérature québécoise Laurent Mailhot (1997 : 199), dans le contexte de ce qu'on appelle «l'âge de la parole", dans l'intervalle de 1948 à 1973, «La poésie rencontre la science, la biologie végétale et animale, dans les " histoires naturelles" de Pierre Morency, ornithologue, auditeur privilégié, et dans les paysages animés, panoramiques ou microscopiques, d'André Ricard (Les Baigneurs de Tadoussac, 1993). Même la poésie du "terroir" prend un coup de (pinceau) jeune, exotique, dans la Beauce française de Grandeur nature ».

Parmi les protagonistes historiques de la poésie québécoise comme Alain Grandbois (1900-1975) - Îles de la nuit (1944) étant une de ses œuvres capitales ou Gaston Miron (1928-1996) - L'Homme rapaillé (1970), pour ne mentionner qu'une de ses œuvres -, une des figures emblématiques de la poésie au Québec est

\footnotetext{
${ }^{4}$ La question n'étant pas centrale dans cette étude, le lecteur intéressé par ces problématiques peut se référer aux travaux de Claude Beausoleil - Le motif de l'identité dans la poésie québécoise (1830-1995) (1996) -, de François Dumont - Usages de la poésie. Le discours des poètes québécois sur la fonction de la poésie (1945-1970) (1993) - ou de Pierre Nepveu - L'Écologie du réel : Mort et naissance de la littérature québécoise contemporaine (1999) -, parmi d'autres.
} 
Paul-Marie Lapointe (1929-2011). Lapointe a occupé un espace central dans la poésie de l'époque et dans la vie politique d'une société qui a connu, à ce moment-là, des redéfinitions et des soulèvements symboliques, car comme l'a remarqué Claude Beausoleil (1996 : 231) «la poésie des années 1970, avec ses expériences langagières poussées aux confins du lisible et de l'illisible, s'était placée dans une position limite : l'écriture, le texte et la forme se donnaient comme univers habitable. Les années 1980 ont été celles de la recherche intérieure, du sujet qui scrute les données de son être ». C'est précisément à partir de cette exploration « du dedans » que les écritures de Paul-Marie Lapointe et Gilles Cyr ont exploré la subjectivité de la relation de l'individu avec l'extérieur. Plus concrètement, avec une visée surréaliste, notamment dans Le Vierge incendié (1948), où l'on trouve (Mailhot, 1997 : 116) « coups de dé, associations libres mais rigoureuses, poèmes-flots, poèmes-crocs ; fenêtres rectangulaires, "irruption volcanique des cours " et des cris, déchirures, scalps, mises à sac. Le "vierge ", c'est la fausse innocence, la peur blême, les limbes sans feu ni lieu », Lapointe a également joué un rôle important dans la construction de la mémoire littéraire à partir du traitement du territoire, particulièrement dans les œuvres Arbres (1960) et Pour les âmes (1964) où l'exploration de l'espace passe par une subversion du langage qui est, néanmoins, profondément informée en matière botanique et géologique ${ }^{5}$. Ici, Lapointe retranscrit et interprète la taxonomie botanique des principales essences de la forêt boréale, en donnant un rythme vertigineux à l'énumération peuplée de nombreuses allitérations des technicismes de la Flore laurentienne - historique inventaire floristique de la vallée du Saint-Laurent « arbitrairement disposées » dans une logique qui n'est pas encyclopédique ni narrative non plus. Pour ce propos, Lapointe se sert d'un manuel intitulé Arbres indigènes du Canada (1950), publié par le Gouvernement fédéral et ratifié par le Service Forestier du Canada, ce qui peut être vu comme une légitimation à la fois scientifique et politique du texte littéraire. Au niveau formel, comme l'a noté Robert Melançon $(1987: 31)^{6}$ :

\footnotetext{
${ }^{5}$ Lapointe aura une influence sur des poètes contemporains à Gilles Cyr, tels que Robert Melançon, qui consacrera une étude au poète intitulé Paul-Marie Lapointe (1987). Notamment, à propos d'Arbres, Melançon (1987 : 27-28) remarque le fait que : «Ce refrain réduit à sa plus simple expression a pour fonction à la fois de scander l'énumération et d'en relancer le mouvement [...] A chaque occurrence il suscite des développements qui rompent l'énumération des essences. Il ouvre ainsi de place en place un espace d'associations libres qui compensent le caractère prévisible de la liste et qui lui confèrent un sens bien autre que celui d'un simple inventaire de la forêt boréale ». Toutefois, Melançon (1987: 31-32) ne trouve pas une revendication nationaliste dans Arbres, car pour lui, « la problématique de nomination et de réappropriation poétique du territoire québécois, qui sera celle de Miron, du Chamberland de Terre Québec, d'Yves Préfontaine, de tant d'autres poètes au cours des années soixante, ne l'intéresse pas dans ce poème ».

${ }^{6}$ Depuis la parution de Peinture aveugle (1978 [réédité en 2010]) la figure de Melançon (Montréal, 1947-) a émergé dans le contexte d'une poésie québécoise en pleine ébullition. Son approche des marges du calme de l'espace urbain, mais surtout de sa délicate attention à l'observation, se nourrit de sa remarquable connaissance de la peinture et des arts. Pour ce poète, traducteur et professeur, l'œil est un organe central ; l'apparition est peut-être un terme approprié pour situer l'un
} 
Ces échappées métaphoriques suivent un rythme complexe, tantôt interrompant l'énumération et se mêlant aux noms d'une famille d'arbres, tantôt la relayant, plus amples, plus développées au début, s'abrégeant peu à peu, plus rapides, elliptiques, pressées lorsque le poème s'approche de sa conclusion, comme dans la strette à la fin d'une fugue le sujet et la réponse se poursuivent avec des entrées de plus en plus rapprochées. Dans son désordre apparent et comme improvisé, Arbres se révèle fermement structuré. Son pouvoir, son charme, tient sans doute pour une bonne part à cet ordre sans raideur, à cette nécessité sans contrainte, à cette liberté sans éparpillement.

Figure proéminente du milieu littéraire, Lapointe était publié aux Éditions L'Hexagone ${ }^{7}$, qui incarnent le contexte littéraire où il a fait émerger avec une force débordante une écriture enracinée dans la terre et la végétation. Cela peut être observé dans l'œuvre Le réel absolu (1971, rééditée en 2014), qui illustre le fait que «L'âge de la parole, du verbe-poème, est d'abord un âge du cri » (Mailhot, 1997 : 110). Il s'agit d'une effervescence sociale qui devient un bouleversement du langage. Comme l'a souligné Laurent Mailhot (1997: 109) dans son étude La littérature québécoise : "Pour Gilles Hénault, Roland Giguère, PaulMarie Lapointe, dès la fin des années quarante, la poésie est mot de passe, revendication, révolte, invasion, éclairage violent. "La poésie authentique est toute semée de molaires qui broient, déchiquettent, décortiquent le langage pour en faire jaillir le sens augural" (Hénault). On est aux " premiers jours du monde", du pays $\gg^{8}$. Rassemblée dans L'espace de vivre (2004) et Le réel absolu (2014), cette poésie fait de son exploration de la physique, de l'astronomie et de la botanique un effort incessant pour chercher des réponses dans le paysage, tout en tissant la neige, le ciel et la montagne pour se rencontrer à l'intérieur, comme des palpita-

des aspects principaux de l'exercice poétique de Melançon. Dans ce cas, la lumière baigne le volume, la forme et favorise une lente et délicate émergence du monde, immergé dans les couleurs. Maintenant à la retraite, Melançon a reçu deux fois le Prix du Gouverneur général : en tant que poète et en tant que traducteur, avec Charlotte Melançon. Il reste à faire une étude comparative mettant en place l'ouvre cyrienne avec celle de Melançon, notamment Peinture aveugle et Le paradis des apparences (2004).

7 À propos de 1'Hexagone Mailhot (1997 : 11) écrit : « Ni chapelle ni école, l'Hexagone est un lieu et un axe de développement, un carrefour ouvert, organisé (en équipe). Il néglige la théorie, les manifestes, pour s'appliquer à produire, à diffuser (par souscription) des plaquettes impartialement choisies. D'abord Deux Sangs, de Miron et Marchand, Des jours et des jours, de Pierrier, etc. La collection «Les Matinaux », destinée aux débutants, se réfère à René Char, qui préfacera Les Cloîtres de l'été de Pilon. Les grands poètes des petites littératures nationales (Césaire, Neruda...) auront aussi une influence décisive. Grandbois, Lasnier, Giguère seront finalement publiés par l'Hexagone, qui, depuis son association avec la Librairie Déom, recueille systématiquement les principaux titres de la poésie québécoise ».

${ }^{8}$ Lapointe, pour sa part, était un des membres fondateurs de la revue Liberté en 1959, et il compte parmi ses distinctions le Prix Gilles-Corbeil 1999, le Prix Léopold Sédar Senghor, le Prix littéraire de La Presse ou l'International Poetry Forum. 
tions. À cette période de l'histoire littéraire du Québec, le poétique, le philosophique et le politique convergent en diverses démarches esthétiques et sociologiques, dont l'intérêt pour la science est fédéré par une enquête qui donne une unité et une cohérence à la pluralité des volontés collectives et individuelles. Laurent Mailhot (1997 : 109) l'a synthétisée ainsi :

Le thème du pays (de la fondation, de l'appartenance), qu'on assimile plus ou moins au mouvement de l'Hexagone, est très différent du régionalisme descriptif et du patriotisme traditionnel, même s'il leur emprunte quelques images. Il s'agit ici d'un projet, d'une «mémoire du futur », d'une cosmo-anthropogonie. Arbres, de Paul-Marie Lapointe, est un inventaire onomastique, une forêt de signes (substantifs), une prise de possession de l'aubier par l'aube, du genévrier par «le plomb des alphabets »: «j'écris arbre », l'arbre pluriel, généalogique et métonymique, l'arbre des livres et des lits. L'automne d'octobre, flamboyant, printanier, s'oppose aux cendres de novembre : «l'agonie des érables t'enseigne le sang de vivre », dit Yves Préfontaine dans Pays sans parole.

C'est dans ce contexte qu'il faut considérer et mesurer l'impact des idées scientifiques sur la poétique. À la fois située et distanciée de ce milieu, l'œuvre de Gilles Cyr montre des particularités qui l'éloignent de la revendication sociopolitique du territoire pour favoriser une exploration plus corporelle de l'espace, où le corps déambulant devient le réseau que reçoit et interprète le monde. En ce qui concerne plus spécifiquement l'écriture de Cyr, le critique Jacques Paquin a abordé, dans son étude intitulée "Variations sur la pente du langage : le poème comme simulacre du raisonnement scientifique» (2003), des aspects de l'interdiscursivité science - littérature, en analysant les notions de « vérité lyrique » et de «vérité scientifique » dans un minutieux travail qui a confronté plusieurs recueils. Concrètement (Paquin, $2003: 32$ ) :

Trois recueils de Gilles Cyr font directement ou indirectement appel à des connaissances scientifiques, à savoir la mécanique newtonienne (Songe que je bouge), l'astronomie (Andromède attendra) et la physique moderne (Pourquoi ça gondole). Cette œuvre poétique nous rappelle une époque où la philosophie se rangeait du côté du savoir (épistémè), alors que la théorie (theoria), quant à elle, relevait du mode du voir.

Paquin récupère la distinction proposée par Dahan-Gaida (1991) entre « intertextualité » et «interdiscursivité » pour analyser la relation entre des savoirs dits «exacts» et d'autres dits « inexacts». Pour Dahan-Gaida, l'intertextualité fait référence aux processus d'interprétation parmi les discours, tandis que «l'interdiscursivité » prête attention à l'interaction entre les principes. Paquin fait un parcours par les tensions savoirs - incertitude ainsi que par les perplexités de ces trois recueils pour affirmer que :

[...] chez Cyr, l'ataraxie est recherchée, tant au niveau du sens que de la syntaxe. Or, on peut se demander si les séries 
causales, en apparence tout à fait indépendantes, n'apparaissent comme telles que parce que le texte ne nous donne pas tous les éléments pour vérifier leur fondement (Paquin, $2003: 36$ ).

L'incertitude, la perplexité et la tension sont en effet des composantes caractéristiques de la poétique cyrienne ; si une connaissance épistémique est ici invoquée, elle est neutralisée par le contexte grammatical qui suspend sa valeur descriptive de modèle de réalité. Son invocation ne possède non plus une qualité nominale, car les concepts ne sont pas suffisamment développés pour générer une narratologie permettant des syllogismes ou des démonstrations. De ce fait, Paquin (2003 : 37) parle d'une «validation à l'intérieur des procédés argumentatifs des poèmes de $\mathrm{Cyr} »$, ce qui suggère qu'un processus de ré-signification articule les concepts scientifiques reliés dans les textes par leur caractère contingent. Comme Paquin (2003 : 37) l'indique :

Il faut lier l'expression du sujet cyrien, qui vise justement à imposer ses propres conditions de vérité, au processus de validation d'une vérité inscrite dans un autre monde de référence. Instituer un mode explicatif du monde et de l'univers, en particulier avec l'usage du si hypothétique, c'est marquer la prise en charge de l'énoncé par le locuteur. Lorsque l'on passe au discours plus proprement scientifique, c'est le soit qui se substitue au si hypothétique et, bien qu'il ait la même signification, il ne vise pas à lever les conditions de vérité, mais au contraire à vérifier la concordance entre un fait et une hypothèse, et à valider l'observation par une expérience.

À titre illustratif, l'on peut mentionner Pourquoi ça gondole (1999), une œuvre peuplée d'annotations astronomiques et botaniques. Ici convergent des remarques provenant de la physique classique, se tournant vers l'ouverture de l'immensité - « en gros j'ai lu / l'univers est un gaz » (9); «Des / galaxies reculent / c'est / la bonne décision » (28) ; «l'infiniment grand / mettons que c'est vulgaire » (14), ou encore « à l'expansion chérie / indéfiniment ralentie » (22)». De même, l'on observe des lectures de la physique quantique dans des formulations concernant l'hermétique et l'exiguïté - «il paraît que la matière / elle n'augmente plus » (19) ; «d'ailleurs les mouvements / locaux deviennent intéressants » (30) ; « l'électron d'avant-hier // est capable de passer / par deux trous en même temps » (31); «l'univers vient du vide / le vide nécessaire » (69) ou encore « l'univers plus petit / que celui de Shapley // sera un peu plus grand / que celui de Kapteyn // ce qui correspondait / à mes propres calculs » (34). Notamment, c'est grâce à la tension syntaxique que des concepts tels que «neutrinos », «électrons », «matière sombre », etc. trouvent un sens renouvelé dans le poème que l'on pourrait qualifier "d'asymétrique ». Voilà pourquoi, comme l'a remarqué Paquin $(2003: 38)$ :

[...] les signifiants, chez Cyr, créent une forme de cohérence que ne peut procurer le rapport à l'espace; sa poésie est 
écartelée même, sur le plan physique, entre l'infiniment grand et l'infiniment petit, sur le plan conceptuel, entre les généralités et la singularité des expériences en cours, non pas à l'intérieur d'un laboratoire, mais au sein du quotidien le plus banal. Le sujet se trouve en quelque sorte à négocier un espace entre ces deux dimensions.

Les premiers recueils de Cyr font déjà allusion à l'espace : Sol inapparent (1978), Diminution d'une pièce (1983), Songe que je bouge (1994). On y trouvera souvent un sujet lyrique qui se déplace en faisant des promenades par la route ; ce mouvement est donc l'origine d'une description de la perception de phénomènes en apparence simples comme l'air, la terre ou la neige. Cette description est, toutefois, incessante mais elle se produit également d'une manière discontinue et interrompue. Cet aspect atmosphérique des premiers recueils de Gilles Cyr est important car cela justifie le potentiel de cette poétique comme objet d'étude de la phénoménologie, de la sémiotique cognitive et, par conséquent, de la poétique cognitive.

\section{Perception et mouvement dans le langage poétique}

L'espace commence par l'image, aussi bien dans la pensée que dans le poème. Le poème est une composition linguistique qui opère avec les rythmes de l'icône en les disposant dans une structure d'interactions dont le contenu perceptif invite à questionner comment une perception peut donner lieu à l'abstraction d'une pensée concrète. Cette abstraction est dirigée par la médiation de percepts les unités de sens du perçu - que l'écriture littéraire dispose tout au long d'une telle composition textuelle, donnant lieu à la reconstruction mentale d'un espace. La manière dont la perception humaine est exprimée dans le poème constitue donc une des questions de poétique à considérer au travers de la théorie de la perception, que ce soit philosophique (voire phénoménologique) ou neuroscientifique. Un examen des divers aspects et du fonctionnement de la perception en tant que processus de la cognition humaine devient nécessaire ; l'objectif, pourtant, est de clarifier les caractéristiques de la pensée poétique au travers de ses représentations du contenu perceptif. Puisque le terme «perception» fait référence à la fois au processus et au résultat cognitif de l'opération qu'il désigne, il est nécessaire d'établir une classification de la visualité en fonction des modèles mentaux du « vu » tels qu'ils sont exprimés par le poème, en termes à la fois de percepts représentés et de procédures de l'expérience perceptive, et dans lesquels il est possible d'identifier les caractéristiques d'une expérience réelle. En effet, la complexité des tâches perceptives accomplies par le système visuel implique la reconnaissance des éléments du réel dans une composition iconique qui apparaît à la fois unifiée, cohérente et dynamique. Il s'agit d'une pensée qui se montre codifiée dans une image unifiée : unité et mouvement ne sont donc pas des propriétés contradictoires par lesquelles les objets sont reconnus par l'observateur, car l'exercice d'identification «du perçu »-le quoi de la perception - est souvent un acte d'association, engageant fréquemment une pré-connaissance desdits éléments. 
Comprendre dans quelle mesure la perception présuppose une pré-connaissance et à quel point elle est source d'un nouveau savoir est une des tâches qu'une proposition théorique sur la pensée créatrice doit affronter. Concept - le trouvé - et percept - le reçu - ne sont pas seulement en tension dans le processus de percevoir mais aussi dans l'espace que le poème délimite. En conséquence, un travail théorico-analytique doit distinguer une taxonomie du regard en fonction des conditions de possibilité du perçu. Pour synthétiser avant de développer, l'on fera allusion à des concepts tels que 1) vision épistémique, 2) vision non-épistémique, 3) représentation mentale, 4) représentations visuomotrices, 5) illusions visuelles et 6) perceptions visuelles. Pour sa part, l'expression littéraire de ces phénomènes cognitifs conduit à établir également une taxonomie des représentations perçues dans le corpus poétique, en tenant compte des notions telles que : 1) visualité et mouvement, 2) visualité et contenu mental et 3) Contemplation du «vide».

Dans son ouvrage interdisciplinaire Ways of Seeing : The Scope and Limits of Visual Cognition (2008 [2003]), Pierre Jacob et Marc Jeannerod exposent l'idée que la perception visuelle est une fonction cruciale de la vision humaine, mais toute vision humaine n'a pas été conçue pour permettre exclusivement la perception visuelle. En fait, - remarquent-ils - une grande partie du système visuel humain est orientée vers le contrôle de l'action, et en particulier le contrôle des mouvements de la main qui sont visuellement dirigés vers des objets. Se préparer à se mouvoir est une des fonctions dérivées de la vision. En outre, Jacob et Jeannerod offrent des éléments empiriques sur le fonctionnement de la perception, notamment, sur la façon dont différents attributs visuels d'un objet sont traités par des aires différentes du système visuel humain. Ils démontrent aussi que le rôle fondamental de la perception visuelle des objets est de permettre l'identification et la reconnaissance des choses : «For the purpose of recognizing the identity of an object, especially for the purpose of identifying the function of an artefact, visual information about it needs to be compared with data stored in long-term memory » (Jacob et Jeannerod, $2008: 140)$, donnant lieu ainsi à la représentation sémantique d'un objet. Bien qu'ancrée dans la mémoire du sujet, cette représentation sémantique de l'objet permet sa verbalisation - voire, sa communication -. En fait, pour Jacob et Jeannerod, la subjectivité et l'objectivité convergent toutes deux dans la perception, qu'elle soit visuelle ou liée à un autre sens, ce qui justifie la valeur de la perception comme moyen de connaissance des objets, faits ou événements :

The puzzle of visual perception is the puzzle of how a purely subjective visual experience can provide us with objective knowledge of the world. [...] visual percepts have nonconceptual content: we examine the paradigmatic arguments from philosophers who appeal to the distinctive phenomenology of visual experience in order to justify the distinction between the conceptual content of thoughts and the nonconceptual content of visual experiences. [....] Not all mental representations need have purely conceptual descriptive content (Jacob et Jeannerod, $2003: 3$ ). 
Dans l'étendue du champ visuel se produisent des interactions qui suscitent l'abstraction des objets par l'observateur, ceci donnant lieu à leur récupération «imaginaire » ultérieure par voie de mémorisation de leurs attributs. Pour Jacob et Jeannerod (2003:20), la perception - processus mental et résultat perçu - rend possibles les représentations mentales, qui se déclinent en plusieurs variétés, telles que des «pensées », « jugements », « croyances », « désirs », « expériences perceptuelles » et aussi des « images mentales ». Cependant, pour la théorie de la perception, la question du surgissement du concept en soi est extrêmement complexe aussi bien d'un point de vue phénoménologique que neurocognitif. Les conditions d'apparition d'un objet dans le champ visuel requièrent, par exemple, que le contenu de la perception visuelle ait une structure spatiale impliquant une perspective, comme l'affirment Jacob et Jeannerod (2003: 21) : «Unlike thought, visual perception has a spatial, perspectival, iconic and/or pictorial structure not shared by conceptual thought. Arguably, one can visually perceive dots with no spatial internal structure, but one cannot visually perceive an object unless it has some spatial location $[\ldots]$ ». Ainsi, dans leur chapitre « The purposes of vision: perceiving, thinking and acting », Jacob et Jeannerod proposent un cadre téléosémantique pour réfléchir à la notion de représentation mentale, en faisant une distinction entre « pensée » et « représentation visuomotrice ». De même, pour eux, les « percepts visuels » (visual percepts) et les représentations visuomotrices (visuomotor representations) sont des représentations mentales qui ne possèdent pas de contenu conceptuel - ce qui sera un aspect fondamental de l'analyse littéraire de cette étude - . Ainsi, pour leur part, les « illusions visuelles » exemplifient la dissociation entre les choses telles qu'elles sont visuellement perçues et les objets construits par la voie du jugement. En conséquence, pour Jacob et Jeannerod les apparences visuelles sont, jusqu'à un certain degré, détachées de la connaissance conceptuelle de la même manière que les jugements diffèrent souvent des apparences des choses. Ceci fait l'objet d'une vaste recherche empirique qui montre qu'un seul stimulus visuel peut donner lieu à un «percept illusoire » et à une « représentation visuomotrice » hétérogènes, dont le contenu abstrait diffère à certains égards du contenu du percept (Jacob et Jeannerod, 2003 : XVI). Cela conduit Jacob et Jeannerod à élaborer une analyse détaillée des deux types de représentations visuelles auxquelles donnent lieu tous les objets qui sont dans le champ perceptif et dans l'espace d'interaction des êtres humains : les «percepts visuels » et les « représentations visuomotrices ». Ainsi :

The former serve as input to higher human cognitive processes, including memory, categorization, conceptual thought and reasoning. The latter is at the service of human action. From the standpoint of our [...] "two visual systems" hypothesis, vision serves two masters: thinking about, and acting upon, the world (Jacob et Jeannerod, $2003: 46$ ).

Dans l'imagination littéraire, les catégories de traitement du perçu se structurent aussi bien dans la pensée que dans l'action invoquant des réalités fondées sur le vécu, le fait (« lo hecho »), et le conçu («was ist gedacht»), ainsi que sur ce 
qui est ressenti («what is felt»). De manière particulièrement dense dans le langage poétique, les interactions du sujet lyrique qui «exécute» les actes et qui aperçoit l'espace sont basées sur des verbalisations provenant des percepts visuels et des représentations visuomotrices qui servent au lecteur comme déclencheurs de son expérience perceptive et visuomotrice propre, ce qui fait que l'acte du poème soit à la fois propre et étranger. L'action qui se produit à l'intérieur du texte est reconnue comme une action propre et comme étrangère, et l'univers visuel invoqué y est familier tout en étant une découverte; cependant la visualité n'est pas entièrement libre dans la pensée abstraite car elle suit la logique de la perception réelle, et qu'en fait, « [...] the content of visual perceptual representations turns out to be both more fine-grained and informationally richer than the conceptual contents of thoughts »(Jacob et Jeannerod, $2003: 21)$. Malgré cela, dans la cognition humaine, les percepts visuels condensent une information perceptive si épaisse que «certaines des choses qui peuvent être pensées ne peuvent pas être perçues » (Jacob et Jeannerod, 2003 : XVII), ce qui nous ramène à la question centrale du processus de formation des concepts. Pour Jacob et Jeannerod, la pensée et construite par des concepts, qui sont à la base de la fonction d'inférer, étant possible de les combiner et déduire par syllogismes des nouvelles pensées. $\mathrm{Au}$ contraire, les percepts visuels possèdent, pour sa part, un contenu qui ne repose pas sur le concept mais sur l'icône, et par conséquent les percepts visuels ont une charge d'information "plus riche et plus fin que le contenu conceptuel des pensées » (Jacob et Jeannerod, $2003: 31$ ). Pour cette raison, la corrélation entre une pensée et un percept visuel comporte un décalage d'information où le percept visuel perd de la richesse en face du concept.

Néanmoins, parmi les propositions théoriques de Jacob et Jeannerod sur le système visuel, la distinction cognitive la plus pertinente pour l'étude de la perception dans le langage poétique chez Gilles Cyr est celle qui s'opère entre la «vision épistémique » et la «vision non-épistémique ». Ainsi, la vision épistémique implique une opération d'association d'attributs perceptifs qui s'enclenche en vue de reconstruire un percept avec de nouveaux stimuli. Par exemple :

$[\ldots]$ one can see a scarlet diamond without seeing that it is a scarlet diamond. Seeing that the displayed object is a scarlet diamond is epistemic seeing. Seeing that an object is a scarlet diamond is coming to believe by visual means that a perceptually salient object is a scarlet diamond. One cannot come to have this belief -hence see that an object is a scarlet diamond-unless one has the concepts scarlet and diamond. There is, however, something it is like to see a scarlet diamond that is different from seeing a red circle or a green lettuce, even for someone who lacks both the concept of a diamond and the concept of scarlet. Seeing a scarlet diamond without seeing that it is a scarlet diamond is non-epistemic seeing. An interesting case of weak epistemic seeing is seeing an object as a scarlet diamond (Jacob et Jeannerod, 2003 : XVII). 
Dans le niveau le plus élémentaire de la connaissance par voie de perception, le fait de regarder un objet permet de déduire un fait $(f a c t)$ qui le concerne, si l'observateur possède une information sur les propriétés de tel objet, son contexte et les éléments avec lesquels il interagit. Au contraire, " $\mathrm{Th}[\mathrm{e}]$ transition from seeing one fact to seeing another displays what we called the hierarchical structure of visual knowledge » (Jacob et Jeannerod, 2003 : 146). D'une telle façon, la vision épistémique primaire contient un fait à partir duquel il est possible de reconnaître un objet dans le champ visuel. Pour sa part, dans le passage de la vision épistémique primaire à la vision épistémique secondaire " on passe d'un fait impliquant une voiture perçue à un fait impliquant son voisin non perçu (qui se trouve être propriétaire de la voiture perçue) » (Jacob et Jeannerod, $2003: 146$ ). Ceci est un aspect crucial de la cognition parce que cette opération mentale permet non seulement d'apercevoir un fait qui implique un objet déjà perçu, mais également de déduire un objet non perçu à partir de la perception d'un fait qui lui contient. Dans ce raisonnement, il est légitime de se demander si la vision épistémique constitue vraiment un type vision ou, au contraire, si elle implique une inférence d'une abstraction vers une autre abstraction. Cependant, la vision épistémique telle que la conçoivent Jacob et Jeannerod a une composante visuelle dans la mesure où la perception permet la justification d'une telle revendication épistémique. L'exemple donné par Jacob et Jeannerod est celui de prétendre savoir que notre voisin est dans sa maison car l'on a vu sa voiture garée dans son allée. Dans ce contexte, l'on confère à la vision le pouvoir de fonder un état de connaissance, malgré le fait que notre vérification n'est pas fondée sur une observation réelle mais sur une inférence et, par conséquent, « the justification for knowing an unseen fact is seeing another fact correlated with the former » (Jacob et Jeannerod, 2003 : 147).

La «vision non-épistémique », d'autre part, survient comme une vision sans inférence : comme on l'a vu avant, elle implique de voir sans reconnaissance contextuelle des attributs : c'est voir le diamant sans savoir qu'il s'agit d'un diamant, ni même ce qu'un diamant est en premier lieu. En conséquence, l'on peut se demander si toute vision non-épistémique ne serait qu'une étape conduisant à une reconnaissance épistémique ultérieure, et on peut considérer dans quelle mesure le percept «survit» dans le champ visuel humain sans passer par un processus de nomination. Dit autrement, la notion d'une vision non-épistémique suggère qu'il est possible de neutraliser l'action du concept lorsque le percept apparait dans le champ perceptif. Comme on le soulignera, le langage poétique offre des exemples singuliers de cette sorte de contemplation dépourvue de tout objectif de connaissance.

Le deuxième aspect théorique et poétique de cette étude concerne non seulement la perception visuomotrice mais aussi la construction de significations qui se produit à travers le mouvement. L'importance cognitive du mouvement tient au fait que la description de celui-ci élucide les structures épistémologiques inhérentes à la conscience kinesthésique ; c'est-à-dire, le rôle du mouvement dans la génération d'un savoir sur le monde qui a pour base la relation du corps avec les 
objets, ainsi que leur apprentissage et l'anticipation des comportements des éléments de l'espace. "We literally discover ourselves in movement », déclare la philosophe Maxine Sheets-Johnstone (2011: 117). Il s'agit, bien entendu, d'organismes animés qui retournent aux choses mêmes, comme l'a formulé la prémisse d'Edmund Husserl («zurück zu den Sachen ») ; ainsi, c'est en interaction avec l'entourage que les êtres humains prennent conscience des capacités et des limitations de leurs corps ainsi que de leur manipulation des objets. De plus, le mouvement, le souvenir du mouvement et la capacité de le simuler mentalement dérivent de la perception, et permettent de développer la faculté de concevoir d'autres mouvements possibles, jamais réalisés auparavant, dans le sens d'une animation. Cela, à son tour, permet de concevoir l'animation de formes inanimés par la voie de l'abstraction des qualités du vivant - comme, par exemple, le cas des marionnettes -. Dans son œuvre The Primacy of Movement Maxine Sheets-Johnstone (2011: 116) attire l'attention sur le fait que :

[...] the awareness of corporeal powers [the awareness of "I cans"] does not (and could not) arise ex nihilo. It arises from [everyday] tactile-kinesthetic activity: chewing, reaching, grasping, kicking, etc. The awareness of corporeal powers is thus not the result of reflective musings, whether with or without language... [and hence is] not a matter of wondering What can I do? On the contrary, the sense of corporeal powers is the result either of moving or of already having moved". In such act $[\ldots]$ corporeal powers give rise to corporeal concepts, fundamental human concepts such as grinding, sharpness, hardness, and so on.

De la même manière que l'œuvre Ways of Seeing propose une théorisation des structures visuelles de l'abstraction conceptuelle, The Primacy of Movement met l'accent sur les aspects kinesthésiques de la pensée abstraite. Les propriétés des objets dont le sujet devient conscient sont des attributs identifiés grâce à l'interaction et à la manipulation physique desdits éléments. La conscience de ces propriétés a, par conséquent, une dimension «primordialement » kinesthésique, comme le souligne la phénoménologue Sheets-Johnstone (2011 : 118) :

In making kinetic sense of ourselves, we progressively attain complex conceptual understandings having to do with containment, with consequential relationships, with weight, with effort, and with myriad other bodily-anchored happenings and phenomena that in turn anchor our sense of the world and its happenings and phenomena.

Dans le cadre de l'étude du langage poétique, la perspective phénoménologique permet d'examiner le rôle des gestes et mouvements dans la conformation de la conscience qu'a le sujet lyrique par rapport à l'entourage auquel il participe, soit comme « acteur» qui altère l'espace, soit comme «senseur» qui se construit soi-même en interaction avec l'espace, car "movement forms the I that moves before the I that moves forms movement" (Sheets-Johnstone, 2011 : 118). Cepen- 
dant, dans ce processus d'acquisition d'une conscience lyrique qui dérive de la réflexion sur le mouvement du corps, le langage poétique, lui aussi, prend une forme. Élucider la manière dont la forme du langage est modulée par la conscience kinesthésique est une des priorités poétologiques.

\section{Les promenades de la parole}

Si prépondérante dans la poétique cyrienne, la représentation du mouvement requiert des paramètres d'interprétation qui permettent de caractériser son impact à la fois sur la forme et sur la nature de cette conscience kinesthésique que l'on a appelée « épistème en mouvement ». Pour cela, l'on décrira les différentes isotopies du mouvement à travers les recueils de Cyr et leurs implications cognitives sur le plan de la perception, en commençant par affirmer que, dans une certaine mesure, la marche est écriture, car elle constitue une transformation du regard qui est à la fois une modulation du langage. La marche possède un rythme qui imprime une temporalité à l'acte de traverser l'espace, bien que l'écriture cyrienne soit moins concernée par l'exploration du temps que par celle de l'espace. Ainsi, par exemple, le quatrième livre de poèmes de Gilles Cyr, Songe que je bouge (1994), témoigne de la pause du mouvement, de l'interruption, de l'acte de s'arrêter qui passe par l'immobilisation de la parole dans la page. Cependant, la verbalisation du corps en mouvement concerne la poétique cyrienne dès son premier recueil, Sol inapparent (1978), une œuvre qui crée des médiations irrésolues entre l'espace et le sujet, et où les sens convergent pour reconstituer un scénario à la fois urbain et paysager. Le recueil commence ainsi ${ }^{9}$.

$$
\begin{aligned}
& \text { Le seuil } \\
& \text { et tout de suite c'est } \\
& \text { - la journée. }
\end{aligned}
$$

Au pas, sans relâche au pas et en route

dans nos pas labourés.

/l

Paille, paille au front.

Le seuil, rien pour nous, est paille.

J'ai couru sur les pierres froides.

(Cyr, $2010: 27)$.

Dessinant une fragmentation de la réalité, ce premier poème de Sol inapparent expose la promenade du sujet lyrique comme un déplacement ardu, entravé

\footnotetext{
${ }^{9}$ Dès son apparition ce recueil a connu une attention remarquable dans le contexte littéraire québécois, ayant produit des critiques dans les revues ; par exemple, celle du poète Robert Melançon qui a publié, dans Le Devoir, une des premières références sur Sol inapparent : « Gilles Cyr, poète de l'essentiel » (1979). Également, celle de Pierre Nepveu « Gilles Cyr : Sol inapparent» (1979).
} 
par l'espace qui est un « seuil » que le sujet doit forcer pour traverser ; la corporalité et la spatialité convergent dans un sol dont la nature n'est pas évidente (route, paille), car le langage esquisse le territoire. Vers la fin, le déplacement passe de la lenteur du début («nos pas labourés ») à l'agilité de la fin («j'ai couru »). C'est un déplacement sans destination claire, mais dont la géographie commence à s'adjectiver («sur les pierres froides »). La déclaration explicite de mouvement apparaît dans le dernier vers, accompagné déjà d'un élément perceptif sur lequel on reviendra plus tard : la température. Pour l'instant, l'on peut suggérer que le sujet traverse un dégel, et les pas sont ralentis par une surface de neige qui empêche la vitesse du mouvement, et plus tard accélérés par une surface glissante : la matière intervient dans le mouvement. Tel que Brouillette (2010:69-70) souligne :

La contextualisation spatiale et dynamique de la parole se situe au cœur d'un projet poétique où l'auteur tente non pas d'insérer des images spatiales dans la langue [...] mais de creuser l'entrelacement de la parole et de l'espace dans la manière même de formuler son expérience individuelle. Celle saisie ne s'opère pas sur le mode de la « capture » $[\ldots]$ qui interrompt le cours du temps en fixant une image, mais plutôt sur celui de la «locomotion », qui permet de prendre part au mouvement des choses sans en suivre obligatoirement le rythme.

Ainsi, dans un deuxième cas, les allusions aux phénomènes sensoriels deviennent également partie-prenante de la structure d'exposition du poème. Une des tensions irrésolues est celle de la conceptualisation de la matière, car cette poétique a précisément pour particularité la corporalité enracinée dans une matérialité qui tend vers l'immatériel ; c'est-à-dire, vers l'expression des traits, des gestes, et des mouvements serrés, délimités, où la nature aussi passe d'une manifestation subtile à une autre plus énergétique. «Se mouvoir chez Cyr, c'est moins révéler cette matière que la parcourir », souligne Paquin (2003:39):

Après,

et après un peu d'air.

La terre forte, inconfortable est fermée à un bout.

Quelqu'un travaille. On entend des coups.

Quand le jour est décoffré, sans image reste la terre.

//

Devant la lumière, devant ce qui la montre. 
La terre dure et simple,

la poitrine

agitée,

soumise aux dons de la terre séparée.

(Cyr, 2010 : 49).

Ici, la notion de « représentations visuomotrices » peut accompagner l'interprétation du texte, car le déplacement du corps s'oppose à l'air. S'il y a mouvement, il apparaît lourd, car la terre se montre «forte, inconfortable » et « fermée », ainsi que «dure et simple». La dimension acoustique acquiert une fonction («On entend ») dans la reconstruction de l'image qui passe ici par des percepts sonores («des coups»). Sur le plan auditif, la perception dans l'ensemble de l'œuvre cyrienne opère par des séquences variables qui établissent une vitesse au rythme du déplacement du sujet lyrique. Cela permet d'anticiper les accélérations et décélérations de l'observateur qui tend à garder à distance les phénomènes acoustiques marginaux qui sont décrits et à fragmenter la sonorité en percepts à la fois isolés, et ambigus ; «Une excitation n'est pas perçue lorsqu'elle atteint un organe sensoriel qui n'est pas "accordé" avec elle. La fonction de l'organisme dans la réception des stimuli est [...] de "concevoir" une certaine forme d'excitation », dirait Merleau-Ponty (2012 : 103) dans sa Phénoménologie de la perception. Malgré tout, la question est d'élucider ce qui est invariant à travers toutes ces variations, en caractérisant la « disposition perceptive » du sujet ; ce que l'observateur préserve de l'expérience perceptive pour la représenter de manière défragmentée, et qui vient caractériser les «qualités transversales » de l'œuvre poétique de Gilles Cyr. À savoir, entre autres : la visualité de l'air, la mobilité de la terre et la mesure du froid.

En effet, dans ce poème, ce n'est pas la lumière mais les conditions de sa visibilité qui intéressent ; le sujet percevant est « devant ce qui la montre » comme acteur qui témoigne de son apparition sans nécessairement l'intérioriser. Les mots « sans image reste la terre » justifieront, plus tard, l'idée d'un percept qui survit sans concept dans le poème. «Epistème en mouvement » dans le poème, lorsque l'espace est reconnu sous condition d'éloignement : la respiration («poitrine ») se trouve «agitée » par l'effort physique de traverser la géographie hostile du paysage froid, qui va soumettre le corps «aux dons de la terre séparée ». Le sujet se voit différencié de l'espace par une distance à la fois organique et géographique ; et dans cette distinction, «Le corps se surprend lui-même de l'extérieur en train d'exercer une fonction de connaissance, il essaye de se toucher touchant, il ébauche « une sorte de réflexion » et cela suffirait à le distinguer des objets [...] (Merleau-Ponty, 2012 : 122). Cette division sujet-espace produit une tension qui va s'accentuer sur le plan formel du poème, où l'on trouve une versification « coupée », en apparence "incomplète », ainsi qu'une absence d'éléments métaphoriques. L'absence de la métaphore constitue une singularité de l'écriture cyrienne qui la différencie d'autres auteurs québécois particulièrement prolifiques à 
cet égard, tels que - pour ne donner qu'un exemple - Rina Lasnier (1915-1997). Comme l'a remarqué Jacques Paquin (2003 : 33) :

Cyr n'est pas un poète de la nomination, il est d'abord et avant tout un syntaxier dans la mesure où les rapports entre les choses importent davantage que le noyautage du sens à l'intérieur de la gangue d'un seul mot ou de la formule. [...]. Les poètes de la nomination sont séduits par la métaphore et l'analogie, figures dont la brièveté de l'énoncé est compensée par une charge sémique plus importante. Chez un syntaxier comme Cyr (mais on pourrait aussi évoquer le nom de Saint-Denys Garneau, qui vient spontanément à l'esprit), plus que l'expression de l'instant, la forme narrative vient accompagner l'immédiateté de l'expérience, à l'encontre de l'effet de sens créé par l'usage de la phrase nominale.

En outre, chez Gilles Cyr, le mouvement présuppose l'établissement constant d'une distance par rapport aux objets de l'environnement du sujet percevant. Formellement, il s'agit d'une distance établie par l'image sans que ce soit une association métaphorique ; si une analogie peut avoir lieu, elle se produit par la juxtaposition de termes en tension. Ainsi, la distance articule les signes en tension de cette poétique qui tend à décrire les phénomènes aperçus sans avoir d'impact audelà du strictement corporel, et sans attribuer des appréciations affectives aux percepts, ce qui n'implique pas d'objectivité. En fait, c'est au sein du traitement subjectif du langage que l'on trouve la valeur du discours poétique comme source de connaissance. La disjonction avec l'espace trouve un exemple dans le poème qui suit de Diminution d'une pièce $(1983)^{10}$ :

La route,

le produit froid de la route.

Nommer la séparation

sépare,

rayonne -

Paysage autre :

où finissent les habitations.

/I

L'obstacle qui dure à ma hauteur,

le pied désordonné qui choisit.

\footnotetext{
${ }^{10}$ Composé de quatre parties, Diminution d'une pièce réunit des poèmes écrits de 1968 jusqu'à 1983. L'énigme de cette œuvre commence déjà dans le titre, car « pièce », au sens principal, indique une partie d'un ensemble considérée comme un tout autonome. Cependant, dans des sens secondaires une pièce est une composition littéraire ou musicale, qui peut être extraite (ou non) d'une œuvre plus large et constituant un tout à elle seule. En troisième terme, une pièce est une œuvre en vers ou en prose destinée à être représentée sur une scène par une troupe d'acteurs.
} 
Nous nous quittons.

Avancer vient du talus, vient longtemps de la terre.

(Cyr, 2010 : 98).

Dans ce poème, l'espace où s'établit la distance est la route. Il s'agit d'une urbanité, « paysage autre ", soutenue par une notion d'obstacle à la mobilité. Ici, il est possible de distinguer deux manières de penser un territoire (et ses effets ou « produits »). La première, comme un sujet qui se trouve plongé au centre du lieu en tant que participant, et qui réagit et interagit à l'intérieur des limites de ce lieu depuis un point de vue particulier (ici, les limitations s'expriment au travers du «pied désordonné qui choisit »). La deuxième consiste à penser l'espace comme une abstraction invoquée au travers des éléments mémorisés, en prenant la distance d'un témoin qui serait impliqué dans la scène d'une manière plus impersonnelle et indépendante des phénomènes qui ont lieu. Ainsi, les percepts visuels des espaces cyriens montrent un écart entre sujet et objet, car :

Pour Cyr, le monde se présente exclusivement en termes d'extériorité : la connaissance du monde qui l'entoure ne peut s'établir que par le recours à des relations causales (même feintes ou factices) entre les phénomènes, qu'ils soient thématisés - présence de phénomènes naturels - ou formels. On songe au behaviorisme, où tout se passe comme si on observait le sujet de l'extérieur, mais que les énoncés d'observation étaient dictés par un je qui ressemble, à maints égards, à un il (Paquin, 2003 : 34).

Au-delà, l'on constate une expression particulière du monde perçu au sein du langage cyrien: le regard du «vide». En effet, une contemplation qui se montre sans contenu épistémique semble se manifester dans les représentations « dématérialisées » de l'espace. Il s'agit d'une visualité qui apparaît « elle seule », comme pensée «abstraite» («abstracted»). Cette tendance à la contemplation qui suspend le temps et fragmente la matière met en relief non seulement un espace aperçu «sans contenu conceptuel », mais également la fonction d'une perception «en elle-même », activité cognitive auto-suffisante qui reçoit les percepts visuels sans en tirer de déduction. Le «vide» et la «distanciation» coincident aussi dans ce poème :

L'horizon

que l'image silencieuse ne nourrit pas.

Parfois le sol, comme, tôt accouru

un plancher d'herbes à chérir,

et l'horizon derechef, maintenant dételé -

//

Le froid reprend, immobile.

Je regarde autour de nous. 


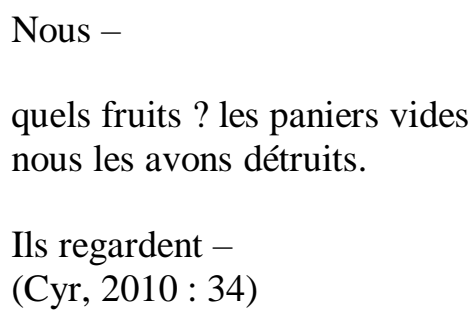

Cet horizon impersonnel apparaît muet pour l'observateur, qui ici se pluralise, en extrapolant cette condition à une perception partagée par «nous » et «ils ». En effet, c'est l'acte de l'observation en lui-même qui devient dans le poème l'objet perçu. L'apparition de l'image dans la distance présuppose à la fois le mouvement du sujet lyrique qui s'éloignait, et une faculté de nomination qui entravait l'association affective. Ainsi, l'horizon va se réduire aux éléments qui le composent. Dans le prochain poème, par exemple, c'est le percept visuel de l'arbre qui apparaît dépourvu de contenu conceptuel :

L'arbre, là-bas : des arbres.

Mais l'arbre seulement.

La lumière qui le trouve,

la bonne lumière n'a pas été dite -

//

L'arbre.

Dans l'hiver qui gêne,

et repose.

L'arbre dans l'hiver imprononçable.

L'arbre

coupé,

la lumière tombe

autrement.

$(\text { Cyr, } 2010: 50)^{11}$

Un autre aspect auquel fait référence la notion poétologique d' « épistème en mouvement ", à travers laquelle on essaie ici d'analyser l'écriture de Gilles Cyr, consiste à souligner comment quelque chose d'éminemment subjectif, tel que l'énoncé poétique de l'expérience perceptive, peut être vu comme une source de savoir vraisemblable sur le monde réel. Comme l'a remarqué Paul Armstrong

${ }^{11} \mathrm{~L}$ 'arbre devient lui aussi une isotopie tout au long de l'œuvre cyrienne, qui contraste avec celle de Lapointe («j'écris arbre»), ainsi qu'avec certains titres de Rina Lasnier : L'Arbre blanc (1966), L'Invisible (1971), Les Signes, (1976), Voir la nuit, (1981) ou L'Ombre jetée (1987). À titre comparatif, l'on peut retrouver un exemple récent dans l'œuvre de Cyr : Huit sorties (2012), divisé en diverses parties dont les titres témoignent de la continuité des isotopies dans la poétique cyrienne sur la perception et l'espace («le mur », «le voyage», «l'arbre», «le jardin», «l'Arménie», «le papillon », «la pomme», «la recherche »). L'extrait suivant est intitulé « ARBRE»: «Tout raser non, d'autant que // botaniste d'un jour / je suis prêt à discuter, moi // le débutant s'approche / roule-t-il des yeux // ne m'interrompez pas / j'aperçois une idée // mousses et lichens / font partie d'un réseau // si les arbres sont rares / adressons-nous aux buissons » (Cyr, 2012 : 40). 
dans How Literature Plays with the Brain. The Neuroscience of Reading and Art (2013), «Vision is information processing, not image transmission, providing "information about what is out there in the world, and how to act on it- not a picture to be looked at" » (Margaret Livingstone, cité par Armstrong, 2013: 58). Mais parce que la vision est aussi «inherently hermeneutic » (Armstrong, 2013: 58), elle est sujette aux nuances des percepts qui, eux, sont biologiques et culturellement construits à partir de l'individu. Ce que nous évoquons, par exemple, par l'aboiement d'un chien - percept à la fois visuel et sonore - diffère d'une personne à l'autre en dépit du consensus sur ce qu'est un aboiement; c'est le récit de cette individualité que le poème offre comme source de connaissance dynamique, car il se base sur l'expérience visuelle :

Given the epistemic importance of visual perception in the hierarchical structure of human knowledge, it is important to understand how by seeing one object, one can provide decisive reasons for knowing facts about objects one does not see (Jacob et Jeannerod, $2003: 148$ ).

Un autre des percepts récurrents de la poétique de Gilles Cyr est l'air. Les images faisant allusion au contact avec l'air font référence à un sujet qui le conçoit comme un obstacle au déplacement. Dans la procédure conventionnelle de l'écriture, la reconnaissance perceptive passe par l'identification nominale d'un terme auquel l'observateur attribue les caractéristiques propres du percept; comme le remarque aussi le poète Jacques Ancet (2019: 27) : «Nous n'avons jamais affaire à des "choses ", mais à des " images ", qui sont, au sens large, des interprétations verbales apprises et immédiatement répétées ». Cependant, la singularité de la poétique cyrienne consiste à opérer avec des percepts dépourvus de contenu conceptuel, dont le logos du paysage phénoménologique est ici kinesthésique, comme on peut le constater dans Sol inapparent : "Cet air, devant la montagne // je sors de là, usé / comme la pierre ou le champ // la montagne se resserre -// (Cyr, 2010 : 29). Usée à cause du contact avec l'air, la montagne aussi perd sa place dans la géographie. Juxtaposition encore une fois, « pierre » et « champ » se déploient dans deux dimensions différentes du paysage " compressé ». Et encore, perception et mouvement sont des actions qu'un observateur exécute en ayant par conséquent un certain type de connaissance :

Vers le sol, vers,

à travers l'empêchement de l'air, cette étendue où tout paraît (herbes, plumes, débris)

sur fond de quelque chose

qui n'est pas le sol lui-même, mais un autre.

//

Sur la terre inconnue, plus loin que l'intervalle de la terre inoccupée j'ai vu, plus loin encore, la terre 
comme un chemin que nous n'avons pas suivi.

Pour le dire je suis venu jusqu'ici.

(Cyr, $2010: 36)$.

Ce «sol-air » vers lequel se déplace avec difficulté le sujet lyrique constitue un percept qui apparaît avec la densité d'un mur. Le vent entrave, et ce faisant, il acquiert une corporalité, tandis que l'inconnu c'est le lointain, ce qui est en dehors du champ visuel. Ici, le mouvement corporel est à la base de l'abstraction des qualités ainsi que de la conformation des concepts eux-mêmes, et de la conscience de certaines facultés corporelles. La localisation du sujet par rapport à l'espace décrit est importante car "any movement has a certain felt tensional quality, linear quality, amplitudinal quality, and projectional quality" (Sheets-Johnstone, 2011 : 123). Et la philosophe précise :

In every general sense, the felt tensional quality has to do with our sense of effort; the linear quality with both the felt linear contour of our moving body and the linear paths we sense ourselves describing in the process of moving; the amplitudinal quality with both the felt expansiveness or contractiveness of our moving body and the spatial extensiveness or constrictedness of our movement; the felt projectional quality with the way in which we release force or energy (Sheets-Johnstone, $2011:$ 123).

Cette idée permet de lire le poème comme un processus de mouvement interrompu, d'effort d'où résulte la conscience lyrique par le biais d'une fragmentation ou «diminution » des qualités du paysage. C'est une véritable réduction de l'espace à son expression la plus élémentaire, comme l'a remarqué Brouillette (2010 : 68), et qui produit un effet ontologique :

[le vent] est chaque fois synonyme d'un mouvement et d'un parcours qui se dessinent franchement dans l'espace. La force du « vent » réside précisément dans sa mobilité, comparativement à celle du froid qui se situe dans sa fixité, et rejoint en cela le sujet dans son désir d'avancer dans l'espace afin d'aviver sa soif de connaissance du monde. Le « vent » n'agit pas constamment sur le corps de celui qui le perçoit, au contraire, il s'en dégage et va au-devant sans attendre d'être rejoint. Ces traits lui donnent une présence que les poèmes tentent parfois de représenter uniquement par la nomination [...]. Ces formulations concentrent l'attention sur l'objet désigné et contribuent à en faire valoir la stricte présence. [...]. Caractérisé à plusieurs reprises par son mouvement de traversée, le « vent» est aussi associé au sentiment d'existence [...]. Peu importe ici que ce soit son action ou son effet, c'est le mouvement lui-même, son énergie et ses capacités de transformation qui révèlent la nature existentielle des choses. 
Finalement, la prépondérance de la basse température dans l'écriture cyrienne met en évidence le rôle de la peau et du système nerveux dans la représentation d'une sensorialité qui passera, cependant, par sa visualité et non, par exemple, au travers de compensations homéostatiques du flux sanguin. Le poème cyrien tend à visualiser les effets du froid sur les choses. Pour insister sur le froid comme perception qui participe du processus de construction d'une conscience lyrique et de son abstraction des qualités du monde, la phénoménologue SheetsJohnstone approfondit la question du mouvement à la première personne du singulier :

$[\ldots]$ if we take seriously that the (experience) "I move" precedes the (conceptual realization) "I can do," and if we take with equal seriousness the fact that specific perceptual awarenesses of ourselves arising in everyday tactile-kinesthetic acts of doing something are the touchstone and bedrock of our discovery of "I cans" and in turn of corporeal concepts, then it is clear that movement is absolutely foundation not only to perceptual realizations of ourselves as doing or accomplishing certain things or making certain things happen -such as "grinding something to pieces"- and to correlative cognitive realizations of ourselves as capable of just such acts or activities, but to perceptual-cognitive realizations of ourselves as alive, i.e. as living creatures, animate organisms, or animate forms. Aliveness is thus a concept as grounded in movement as the concept "I can" (SheetsJohnstone, 2011: 116).

Ainsi, en définitive, un dernier extrait de Diminution d'une pièce contient une expression où vent, main et bouche convergent dans un geste qui exprime l'incarnation de l'interaction corporelle avec l'inconnue :

Dehors je reçois les dehors.

La main dure,

la terre sèche

comme le vent s'en va,

la main sèche qui n'a rien vu.

/I

La montagne, à pied.

Lorsque le froid va s'opposer

ici, avec des frappements

quelle bouche tendue,

pour apercevoir le froid -

(Cyr, $2010: 70)$.

Le mouvement étant un facteur de réchauffement du corps, on observe dans la convergence du vent froid, de la main et de la bouche, le geste d'une con- 
traction musculaire qui ralentit le déplacement. L'interaction est limitée par les caractéristiques météorologiques du paysage qui opèrent ici comme des tenseurs ayant des répercussions non seulement sur la tension du visage et des mains, mais également sur l'assimilation cognitive de l'environnement que le mouvement favorise : «Le froid, / la terre // le nouveau froid // il trouve / et déferre / les portes qui n'ont pas interrompu - , dit Gilles Cyr (2010 : 75). Ainsi, le froid et la terre accompagnent l'immobilisation du corps et de son récit lorsque le verbe transitif « déferrer » entre dans le champ sémantique de la limitation du mouvement, de même que ces «frappements » suggèrent une alternance de mouvements serrés, dont l'expression grammaticale est ralentie : le froid réduit le mouvement dans le poème. Ainsi, l'isotopie du froid nous invite à développer d'autres recherches, dont ferait partie l'étude de la sensorialité du discours poétique analysée au sein d'un cadre théorique sur la perception de la température. Comme on l'a montré, la « visualité » du froid interpelle ces poèmes, où la température parait jouer un rôle crucial dans la concentration mentale. C'est ainsi que les textes cyriens créent un espace d'incertitude du corps qui se déplace.

Au long de cette étude, on a fait allusion à des visions épistémiques et nonépistémiques, ainsi qu'à des représentations visuomotrices, des illusions visuelles et des perceptions visuelles : opérations cognitives du cerveau qui sont projetées dans le niveau sensible du poème cyrien. L'expression littéraire de ces phénomènes cognitifs nous a conduits à suggérer une taxonomie des représentations perçues dans le corpus poétique, en tenant compte des binômes tels que visualité et mouvement, visualité et contenu mental, contemplation $d u$ "vide », et visualité et température, dans l'abstraction de connaissances du sujet lyrique. En effet, la notion d'une «épistème en mouvement» peut en élucider, dans de futures recherches, les processus cognitifs favorisés par les gestualités du corps et leur implication dans la conformation d'une conscience spatiale dans le sujet. Une telle tâche aurait pour objectif une connaissance plus approfondie des stratégies du langage poétique afin d'exprimer l'abstraction des savoirs qui caractérise l'imagination chez l'être humain.

\section{RÉFÉRENCES BIBLIOGRAPHIQUES}

ANCET, Jacques (2019) : Amnésie du présent. Paris, Éditions Publie.net.

Armstrong, Paul B. (2013) : How Literature Plays with the Brain. The Neuroscience of Reading and Art. Baltimore, Johns Hopkins.

BERMÚDEZ, Víctor (2015) : «Lorand Gaspar et la poésie-épochè vers une connaissance du doute». Lorand Gaspar et la matière-monde, Marie-Antoinnette LaffontBissay \& Anis Nouairi (eds.). Paris, Éditions L'Harmattan, 103-125.

BERMÚDEZ, Víctor (2017) : Ciencia y modulación del pensamiento poético: percepción, emoción y metáfora en la escritura de Lorand Gaspar. Salamanca, Ediciones Universidad de Salamanca. 
BERMÚDEZ, Víctor (2019) : «Aprender en la incertidumbre: entrevista a Gilles Cyr ». Humanidades: revista de la Universidad de Montevideo, 6, 283-288.

BRouilletTe, Marc André [dir.] (2003) : [Dossier] Gilles Cyr. Voix et Images, 84, 5179.

BrouilletTe, Marc André (2010) : Spatialité textuelle dans la poésie contemporaine. Gilles Cyr, Jean Laude et Anne-Marie Albiach. Québec, Éditions Nota Bene.

CYR, Gilles. Poèmes 1968-1994 (2010) : Préface de Marc André Brouillette. Montréal, Éditions Typo.

CYR, Gilles (1970) : «Littérature et science ». Liberté, 12:1, 57-67.

DAHAN-GAIDA, Laurence (1991): «Du savoir à la fiction: les phénomènes d'interdiscursivité entre science et littérature ». Revue canadienne de littérature comparée, XVIII:4, 471-487.

DUMONT, François (1993) : Usages de la poésie. Le discours des poètes québécois sur la fonction de la poésie (1945-1970). Québec, Presses de l'Université Laval.

JACOB, Pierre \& Marc JEANNEROD (2003) : Ways of Seeing: The Scope and Limits of Visual Cognition. Oxford, Oxford University Press.

MAILHOT, Laurent (1997) : La littérature québécoise. Montréal, Éditions Typo.

MaIlhot, Laurent et Pierre NePveu (1996) : La Poésie québécoise. Des origines à nos jours. Montréal, Éditions Typo.

Melançon, Robert (1979) : « Gilles Cyr, poète de l'essentiel », Le Devoir, 3, 19.

MELANÇON, Robert (1984) : «Gilles Cyr, poète du continu ». Liberté, 153, 159-160.

MElançon, Robert (1987) : Paul-Marie Lapointe. Paris, Éditions Seghers.

MERLEAU-PONTY, Maurice (2012 [1975]) : Phénoménologie de la perception. Paris, Gallimard.

NEPVEU, Pierre (1979) : «Gilles Cyr : Sol inapparent», Livres et auteurs québécois 1978, Montréal, Éditions Jumonville, 112-113.

NEPVEU, Pierre (1984) : «Écriture-tatouage et langage raréfié ». Le Devoir, 31, 22.

PAQUIN, Jacques (2003) : «Variations sur la pente du langage : le poème comme simulacre du raisonnement scientifique », Voix et Images, 84, 31-43.

PAQUIN, Jacques (2014) : Anthologie Science et Poésie. Trois-Rivières, Écrits des Forges.

SheETS-Johnstone, Maxine (2011) : The Primacy of Movement. Amsterdam, John Benjamins Publishing Company. 